\title{
BMJ Open How to improve emergency care to adults discharged within 24 hours? Acute Care planning in Emergency departments (The ACE study): a protocol of a participatory design study
}

\author{
Christina Østervang (D) ,1,2 Annmarie Touborg Lassen (D) ,,2 \\ Charlotte Myhre Jensen (D) ,2,3 Elisabeth Coyne (D) ,2,4 \\ Karin Brochstedt Dieperink (i) ${ }^{2,5}$
}

To cite: Østervang C, Lassen AT, Jensen CM, et al. How to improve emergency care to adults discharged within 24 hours? Acute Care planning in Emergency departments (The ACE study): a protocol of a participatory design study. BMJ Open 2020;10:e041743. doi:10.1136/ bmjopen-2020-041743

- Prepublication history for this paper is available online. To view these files, please visit the journal online (http://dx.doi. org/10.1136/bmjopen-2020041743).

Received 17 June 2020 Revised 27 November 2020 Accepted 05 December 2020

D) Check for updates

(C) Author(s) (or their employer(s)) 2021. Re-use permitted under CC BY-NC. No commercial re-use. See rights and permissions. Published by BMJ.

For numbered affiliations see end of article.

\section{Correspondence to} Mrs Christina Østervang; christina.oestervang.nielsen@ rsyd.dk

\section{ABSTRACT}

Introduction The development of acute symptoms or changes in diseases led to feelings of fear and vulnerability and the need for health professional support. Therefore, the care provided in the acute medical and surgical areas of the emergency department (ED) is highly important as it influences the confidence of patients and families in managing everyday life after discharge. There is an increase in short-episode ( $<24$ hours) hospital admissions, related to demographic changes and a focus on outpatient care. Clear discharge information and inclusion in treatment decisions increase the patient's and family's ability to understand and manage health needs after discharge, reduces the risk of readmission. This study aims to identify the needs for ED care and develop a solution to improve outcomes of patients discharged within 24 hours of admission.

Methods and analysis The study comprises the three phases of a participatory design (PD). Phase 1 aims to understand and identify patient and family needs when discharged within 24 hours of admission. A qualitative observational study will be conducted in two different EDs, followed by 20 joint interviews with patients and their families. Four focus group interviews with healthcare professionals will provide understanding of the short pathways. Findings from phase 1 will inform phase 2, which aims to develop a solution to improve patient outcomes. Three workshops gathering relevant stakeholders are arranged in the design plus development of a solution with specific outcomes. The solution will be implemented and tested in phase 3 . Here we report the study protocol of phase 1 and 2 .

Ethics and dissemination The study is registered with the Danish Data Protection Agency (19/22672). Approval of the project has been granted by the Regional Committees on Health Research Ethics for Southern Denmark (S-20192000-111). Findings will be published in suitable international journals and disseminated through conferences.

\section{INTRODUCTION}

When patients have an acute episode of symptoms or instability of a chronic disease, they
Strength and limitations of the study

The proposed study will, through participatory design (PD), combine methods into the design and test of an innovative solution, seeking to improve patient and family outcomes in connection to their discharge from the emergency department (ED). This will provide insight into patient and family needs during their ED pathway.

- It is a key feature in the study to ensure user involvement from all stakeholders and sustainability of the developed solution, as it is drawn directly from patients', family members' and healthcare professionals' statements, experiences and ideas.

- The study includes family perspectives, which is limited in previous research from an ED perspective.

- Using PD could be time-consuming and might be a limitation, as it could be difficult to gather relevant stakeholders at the same time.

often have feelings of fear and helplessness due to the uncertainty of the situation. This brings patients and their families to the emergency department (ED) in a vulnerable and distressed situation. ${ }^{1}$ The care provided at the ED will influence the patient's and family members' experience of the current stay and influence their ability to understand and use health information for maintaining their health after discharge. ${ }^{1-3}$ Family members rank supportive communication with nurses as vital to reduce stress and anxiety. ${ }^{4}$ Emergency nursing care is administered by systematic guidelines based on, for example, Airway, Breathing, Circulation, Disability, Exposure (ABCDE) principles to support effective patient pathways and to identify specific patient needs, making it possible for nurses to respond rapidly and effectively. ${ }^{5}$ The majority 
of patients with acute symptoms are initially cared for in a general ED or common acute medical and surgical emergency unit. ${ }^{6}$ Many countries have this organisational structure and systematic approach to ensure fast, systematic and comprehensive assessment along with the improvement of patient flow. ${ }^{78}$ The organisational structure has a positive effect on preventing overcrowding and is also a result of the reduced number of in-hospital beds. ${ }^{9}$ Attention is often on organisational concerns, but there is a need for exploring patient-related aspects as well.

Acute nursing care is characterised by rapid and efficient treatments. This often results in short and fragmented encounters between patients and nurses. ${ }^{2} 10$ Previous research on patient perspectives has shown that patients feel that ED nurses seem to lose interest in the patient's life situation after the most acute treatment has been initiated. ${ }^{11}$ In line with this, a Danish National Survey revealed that $33 \%$ of patients did not experience that their family's perspective was considered important. ${ }^{12}$ Furthermore, $30 \%$ of the patients participating in this survey reported that they were not involved in the decision-making process of their care. ${ }^{12}$ These findings indicate that the international and national health standards for patient involvement are not met. ${ }^{1314}$ Healthcare professionals' acknowledgement of the family's role and inclusion in care decisions enable the family to improve the patient outcomes, but also ensure that family caregivers understand information and are able to coordinate care and manage practicalities. ${ }^{15} \mathrm{~A}$ way to improve the quality of care would be to give patients and families a stronger voice. This could help identify their needs and the resources they use, to enable supportive care to be tailored. ${ }^{16}$ To enable nurses to assess and partner with patients and families to meet their needs and tailor care during short nurse-patient interactions, a nurse-led intervention may be useful. ${ }^{17}$ Previous research exploring ED patients' expected outcomes identified four main concerns: understanding diagnosis, symptom relief, reassurance and treatment plans. ${ }^{6}{ }^{18}$ However, the family perspective was not reported in these studies. ED nurses highlight family members as an important resource to obtain information, and needs more research. ${ }^{19}$ Furthermore, research has identified numerous discharge interventions and strategies to prevent readmissions; however, these are primarily concerning elderly, frail patients and not inclusive of family members. ${ }^{20-23}$ Sparse research has been conducted focussing on the diversity of ED patients and their families, highlighting the need for interventions on how to assess and tailor care. ${ }^{24-26}$

\section{Objective}

The overall aim of this study is to improve patient outcomes by nurse assessment and tailoring care for patients and family members discharged from the ED $<24$ hours.

Following research objectives will guide each phase:

1. To create knowledge about what patients, family members, and healthcare professionals do and what they say they do, in connection to patients discharged within 24 hours (phase 1a).

2. To assess the needs and preferences of patients and families admitted in the ED to gain an understanding of patients and family needs (phase $1 \mathrm{~b}$ ).

3. To understand how healthcare professionals in the ED perceive patients and family needs and preferences, and how they would accommodate these in their care (phase 1c).

4. To design and develop a solution to improve patient outcomes using focus group workshops (phase 2).

\section{Methods}

The overall research design and methodology for this study is participatory design (PD) ${ }^{27}$ The Family System Theory $^{28}$ and the framework of Medical Research Council ${ }^{29}$ for developing interventions in healthcare are used to guide the study.

\section{Study design}

PD is chosen as research methodology as it includes the participants in the design phase and is relevant to use in research areas with limited knowledge. ${ }^{27} \mathrm{PD}$ is defined by making innovative solutions to problems in real life through a democratic stance and genuine participation of all relevant participants which represent future end-users of the field. ${ }^{30}$ It enables the focus to be on future endusers in designing an intervention strategy that provides possibilities to improve patient outcomes in the ED. A PD process conducted in health science is typically performed in three interdependent phases ${ }^{31}$ and is characterised by collective 'reflection-in-action' iterations. In phase 1 , the focus is to identify user needs. In phase 2 , a prototype as a solution to cover the identified needs is developed. Finally, the solution is implemented and tested in a clinical setting and its effect and success will be evaluated. Here we report on the study protocol for phase 1 and 2. As the three phases are interdependent, phase 1 will provide the information and inform phase 2 and so on. Therefore, phase 2 cannot be predesigned, wherefore an exploratory approach will be used as design. ${ }^{27}{ }^{32}$ With an explorative approach, patient outcomes are not defined in advance but will be identified by the patients and family members in the initial phase of the study. However, the main outcome must be focussed on the quality of care expressed by patients. A literature review exploring ED patients' outcomes and clinical interventions will be completed for each phase to ensure an understanding of current research to inform the study. ${ }^{33}$

To identify patient and family needs and preferences, field observational studies inspired by Spradley $^{34}$ will be obtained by the first author, followed by joint semistructured interviews of patients and family members. ${ }^{35}$ Focus groups of healthcare professionals will enable sustainable and an achievable solution to develop. An intervention plan developed from phase 1 will be constructed and relevant stakeholders and future end-users of the solution will be invited to participate in three workshops 
Acute Care planning in Emergency departments (The ACE study): protocol of a participatory design study.

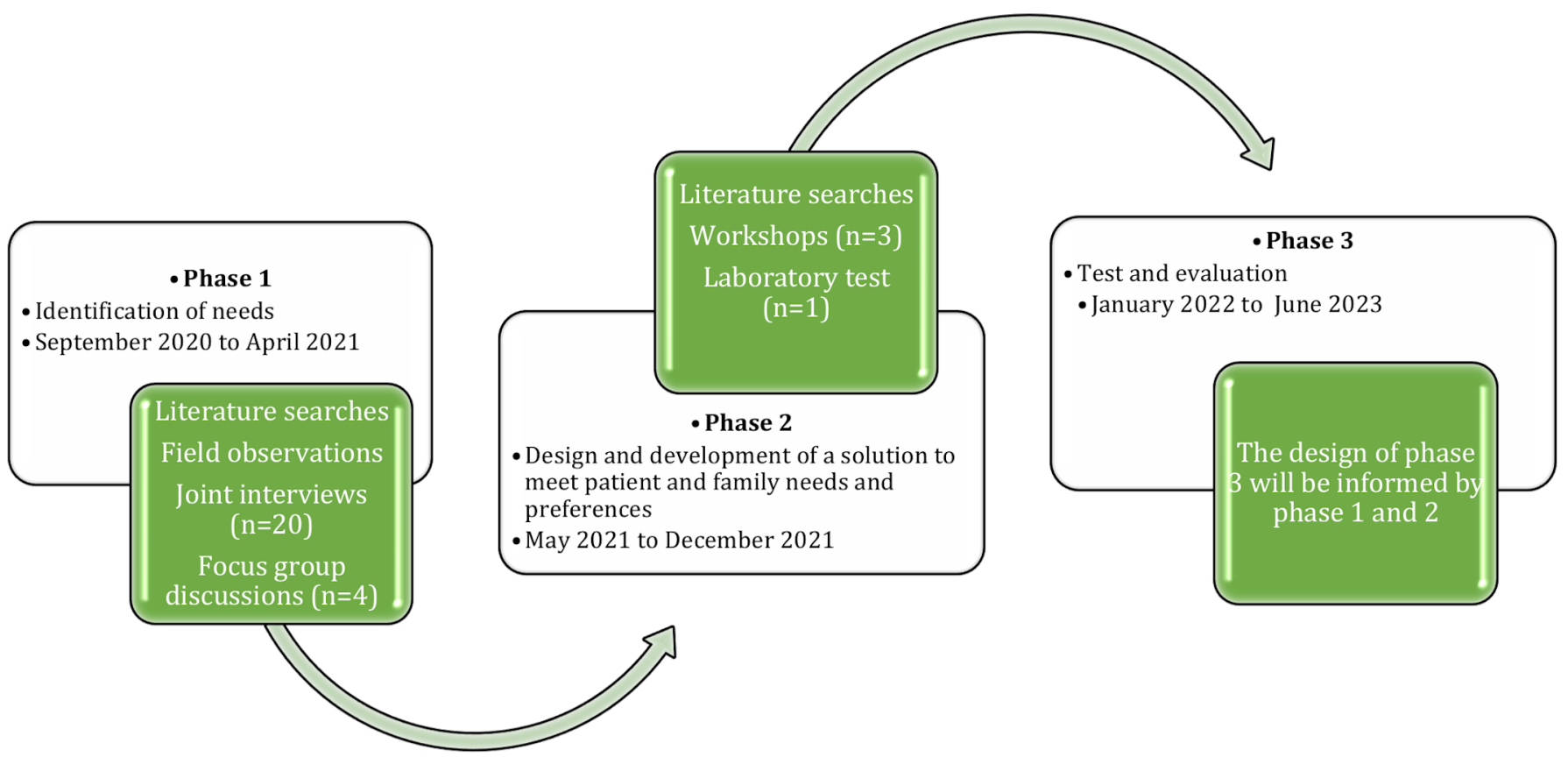

Figure 1 The estimated time frame and methods of the Danish study 'Acute Care planning in Emergency departments, (The ACE study)'.

to finalise the design. The workshops will be designed to focus on: (1) generation of ideas (2) workshop with the intention to create mock-ups for the creation of a final prototype and (3) a 'laboratory' workshop where this prototype is pretested in a clinical setting. ${ }^{27} \mathrm{~A}$ 'laboratory' workshop is characterised as deliberately staged activities during which a controlled environment for exploration is created, and open collaboration between the participants is facilitated. ${ }^{27}$

The Medical Research Council ${ }^{29}$ framework of developing complex interventions will be used to guide this study: (1) development (2) feasibility and (3) evaluation in line with the three phases of the study's research design, as illustrated in figure 1. The Medical Research Council argues that an intervention is complex when it contains several interacting components. ${ }^{29}$ The current study will include a range of patients, families, healthcare professionals and organisational changes.

\section{Theoretical framework}

The theoretical framework is based on the Family Systems Theory $^{28}$ that care is provided holistically with patient and family as the unit of care. According to Wright and Leahey, family members could be spouses, partners, adult children, friends or others from the care-recipient's social network who care for the patient. Family Systems Theory aims to help families to achieve stability in their lives by focusing on their internal relationships, resources and capacity to adapt to new situations caused by illness. ${ }^{28}$
This framework guides the research process including sampling, designing intervention and research aims. After episodes of care in emergency, the family is the main carer and provider of support. Therefore, to improve patient outcomes, the family's inclusion is required to enable family information needs to be met. ${ }^{11}$

\section{Setting}

The study is carried out from September 2020 to June 2023, as shown in figure 1. Data will be collected from the ED at two hospital sites: (1) The Odense University Hospital (OUH), which is a 1000 bed university hospital, and covers all specialities and provides care for a population of 230000 adults living in four municipalities. The ED seeing 69000 annual attendees, mean age 45, treats 180 patients per day with a capacity of 42 beds and 30 examination rooms. On average, 32 patients are admitted to the hospital per day, and $50 \%$ are discharged within 24 hours.

(2) Department of Emergency Medicine, Hospital of Lillebaelt, Kolding. The Hospital of Kolding has the capacity of 320 beds. The ED seeing 50000 annual attendees, mean age 45, receives 146 patients per day and has 58 beds and 5 trauma rooms beds capacity. The EDs are organised as they can control the allocation of the in-hospital beds at the rest of the hospital.

The Danish healthcare system is provided with open access and people do not need health insurance to be seen by a physician as it is a tax-funded welfare system. 
Table 1 Patient features in phase 1 of the Danish study 'Acute Care planning in Emergency departments (The ACE study)'

\begin{tabular}{ll}
\hline Patients $\mathbf{( n = 2 0 )}$ & Specific attributes \\
\hline Age & $\geq 65$ years of age/ $\leq 65$ years of age \\
Sex & Equal male and female \\
Symptoms & Equal surgical/medical symptoms \\
Education level & Below/above secondary school \\
Function level & $\begin{array}{l}\text { Receiving primary care/not receiving } \\
\text { primary care }\end{array}$ \\
Social status & Living independently/living with someone \\
\hline
\end{tabular}

Acute patients are evaluated in person or by emergency calls by primary care physicians who act as gatekeepers before entering the ED. Denmark has a well-established and free of charge primary care, public pre-hospital emergency transport and treatment at public hospitals. When patients are discharged, they can get uncharged follow-up by their general practitioner, primary nursing care or in an outpatient clinic.

The study is affiliated with the Family Focused Healthcare Research Center (FaCe) at the University of Southern Denmark. ${ }^{36}$

\section{Participants}

Patients and family members

\section{Inclusion criteria}

Purposive sampling of patients: $\geq 18$ years of age, Danishspeaking, discharged $<24$ hours with medical or surgical symptoms. Family members, invited by the patient, are included.

The target study population is shown in table 1 .

Sampling strategy will ensure equally represented patients with first time visits among patients with multiple ED visits. Other collected variables: gender, age, civil status, educational level, length and frequency of stay, diagnosis, Charlsons comorbidity score and family relations.

\section{Exclusion criteria}

Cognitive impairment assessed by the nurses by using Glasgow coma scale added by individual clinical judgement according to be able to understand the terms of participating in a research study. Highest and lowest triage level according to Danish Emergence Process Triage. ${ }^{37}$

\section{Healthcare professionals}

Nurses, physicians and physiotherapist working at the $\mathrm{ED}>6$ months will be included. Inclusion will be done purposively to enable a broad sample of healthcare professionals.

Other collected variables: gender, age, profession, years since graduation years of employment at the ED and educational level.
Collaborators and consultants

The participants in this category will be identified during the analysis of phase 1 . It seems relevant to look into previous research, consulting experienced researchers within PD and looking into exciting interventions in healthcare, IT software engineers, design schools, communication advisors, sociologists, anthropologists and cross-sectoral partners.

\section{Phase 1a: field observations \\ Research objective}

To create knowledge about what patients, family members, and healthcare professionals do and what they say they do, in connection to patients discharged within 24 hours.

\section{Method}

Field observations will be conducted in both EDs (estimated $n=10$ days of 4 hours a day) to include relevant perspectives in the understanding of patient and family needs and preferences. We chose four to 6 hours as time frame for the field observations based on National standards stating that patients in the Danish EDs should receive a treatment plan within 4 hours. ${ }^{38}$ All sample sizes in the study are based on scientific guidance of qualitative research ${ }^{39}$ Field observational studies are chosen as it has the strength to create direct knowledge about what participants do and what they say they do, ${ }^{40}$ in connection to their treatment and care in the ED. Field observations are planned at different weekdays and times of the day to show the potential diversity. The duality of being a researcher, experienced nurse and employed at the department at the same time will be accessed as objectively as possible by using a template for documentation of field notes, inspired by Spradley. ${ }^{34}$ Each day, field notes will be taken and transcribed immediately to secure correct recall. ${ }^{34}$ The notes are expected to consist of descriptions, illustrations and short quotations. Approval from the management of the departments was obtained in February 2020. Data from field observations will actively be used to understand what the patients have experienced and inform the development of the interview guide.

The interviewer is an experienced emergency nurse with a Master's degree (12 years of emergency nursing). From previous research, she has experience doing intervention and qualitative research. ${ }^{41}{ }^{42}$ She is supervised by an experienced research team that is involved in every aspect of the project.

\section{Phase 1b: interviews with patients and family members Research objective}

To assess the needs and preferences of patients and families admitted in the ED to gain an understanding of patients and family needs.

\section{Method}

Guided by a phenomenological hermeneutical framework, patients and family members from both EDs will be interviewed face-to-face or by telephone within the first week after their emergency visit $(n=20)$. Recruitment of 
patients and family members will occur during the observational study. Patients will be approached and provided with a plain language information sheet of the study and asked if they would be interested. Once patients are recruited, family members will be invited into the study. Using a purposive sampling technique will ensure balance across the different patient features from table 1.

Semi-structured family interviews will be conducted in person. The interview guide will begin by asking participants to share about their visit to emergency. The researcher will ask participants to elaborate on different aspects of their emergency visit from the observation data collected. Interviews will be conducted at a time and place convenient for the patient and family member. Interviewing patients and family members is aimed at identifying both their individual and common experienced needs and preferences. Interviews enable the participant's perspectives and experiences to be shared to gain an understanding of the experience. ${ }^{43}$ A question example is: "What have you talked about since discharge?' We will continue recruitment until thematic saturation is reached; the point at which no new themes are emerging. ${ }^{39}$ This will include a minimum of 20 participants to secure maximal variation of the target group but will be continued if the thematic saturation is not reached within this sample size. We chose this sampling strategy as it is designed to ensure that a full range of themes is elicited within each group.

\section{Phase 1c: focus group interviews with healthcare professionals}

Research objective

To understand how healthcare professionals in the ED perceive patients and family needs and preferences, and how they would accommodate these in their care.

\section{Method}

Four focus groups will be conducted with approximately $n=20$ nurses and physicians equally from both sites. Focus groups are an effective way to produce group-level data, based on the interpretation, interaction and norms of social groups. ${ }^{44}$ Participants are asked to discuss quotes from patients' and family members' interviews to understand healthcare professionals' perspectives and reactions to these quotes. The interactions between participants can lead to participants contributing spontaneous statements about the given subject, and new ideas are created. The first author moderates the focus group together with one of the more experienced researchers from the research team. Observations of the non-verbal communication, the group-interaction and elaborating questions will be recorded as field notes. ${ }^{44}$ Each focus group will consist of four to six participants. ${ }^{45}$

\section{Analysis: phase 1a-c}

Qualitative data from the joint interviews, focus group interviews and field observational studies will be synthesised and analysed in a phenomenological and hermeneutical framework. The hermeneutic approach allows us to gain an insight into the individual's lived experience and provides an interpretive perspective to explicate meanings and assumptions in the data by studying and interpreting narrative. ${ }^{39}$

To organise the process of the analysis, the steps from Malterud's ${ }^{46}$ systematic text condensation (STC) will be used in NVivo12. First, we will capture a general impression of the data and extract preliminary themes. Second, the data will be allocated into meaningful units which is a text section that represents pieces of information about a research question. The meaningful units will be condensed and coded, and finally, findings will be synthesised. To ensure the trustworthiness and rigour of the analysis process, we will follow the standards for reporting qualitative research of O'Brien et al. ${ }^{47}$

The progressive process line in phase 1 is shown in figure 2 .

\section{Phase 2: design and development of a solution in a workshop process}

The second phase is the actual development of a solution to improve patient outcomes by nurse assessment and improved tailored care to patients and family members, discharged from the ED $<24$ hours.

\section{Research objective}

To design and develop a solution to improve patient outcomes using focus group workshops (phase 2).

\section{Method}

A co-design framework will be used. The process of design and development of a solution will be affected by involving participants across all areas in workshops and

\section{Acute Care planning in Emergency departments (The ACE study): protocol of a participatory} design study.

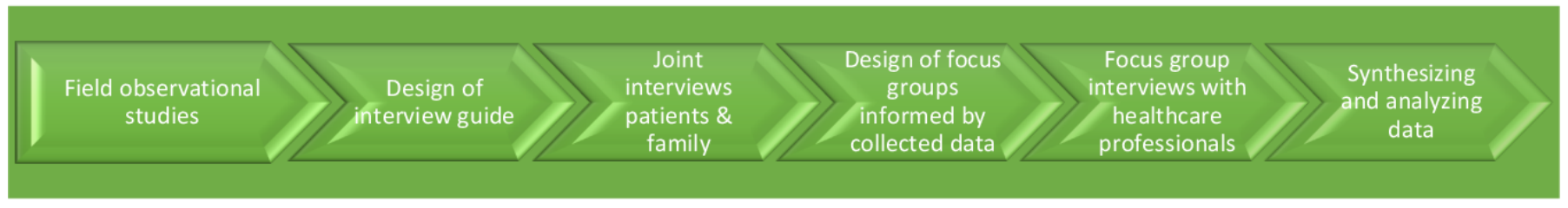

Figure 2 Progressive process of phase 1. 
in the laboratory workshops. This will enable discussion of needs, mutual learning and creativity, ensuring that the solution is innovative and user-focussed. ${ }^{27}$ Initially, an idea-generating workshop will be conducted, followed by a mock-up workshop, creating a temporary prototype of the solution. Workshops will consist of different participants representing different perspectives: patients, family members, various healthcare professionals, IT designers, innovation consultants, the research team among others. Collecting a broad variety of participants with different backgrounds, and perspectives will bring nuanced perspectives to the process and the ability to predict possible challenges with the prototype. ${ }^{27} 29$ The workshops will be facilitated as a space for creativity and 'reflection-in-action' among participants. To facilitate this creative space, visualisation tools will be used, such as posters, personas and note paper or post-it notes. ${ }^{30}$ The use of creative space allows participants and researchers to work as equal partners, bringing the iterative process into action. The results of the analysis will be presented for the invited participants by the research group to create direction. After the initial workshop, the research team will include the relevant stakeholders to proceed with the development of the solution. A possible solution will be informed by study 1 and the workshop process. Looking into previous research, intervention examples could be telehealth solutions, discharge follow-up or cross-sectoral collaboration. $^{48}$

Finally, a 'laboratory' workshop pretesting the prototype sees its feasibility and acceptability in practice. ${ }^{30}$ This workshop will include a smaller number of participants as the aim is narrow, compared with the creative, innovative workshops.
The number of workshops and its attendees will depend on the process, but based on previous research using $\mathrm{PD},{ }^{30} 48$ at least three workshops are estimated.

\section{Analysis}

Data from the workshops will be obtained as pictures, notes on posters, debriefing and recorded discussion during the workshops. The first author will transcribe and systematise the data into themes inspired by STC $^{46}$ and present them as a report. The report will be discussed by the research team and relevant collaborators for final adjustments before the test phase. The analysis and development of the model will be conducted iteratively in the following steps: plan, act, observe and reflect. This process is illustrated in figure 3 .

The phase three evaluation will be developed from the most important patient reported outcomes identified in phase one and targeting the intervention in phase 2 . The evaluation phase three will be published in a separate study protocol.

\section{Data management plan, ethics and dissemination}

Oral and informed consent will be obtained after providing plain language information. ${ }^{49}$ Participation is voluntary, and it is possible, at any time, to withdraw from the study. The study is registered with the Danish Data Protection Agency (19/22672). Approval of the project is obtained from the Regional Committees on Health Research Ethics for Southern Denmark (S-20192000-111).

Data will be stored at Open Patient data Explorative Network (OPEN_938). ${ }^{50}$ Findings will be published in suitable journals and disseminated through workshop and conferences.

\section{Acute Care planning in Emergency departments (The ACE Study): Protocol of a participatory}

\section{design study}

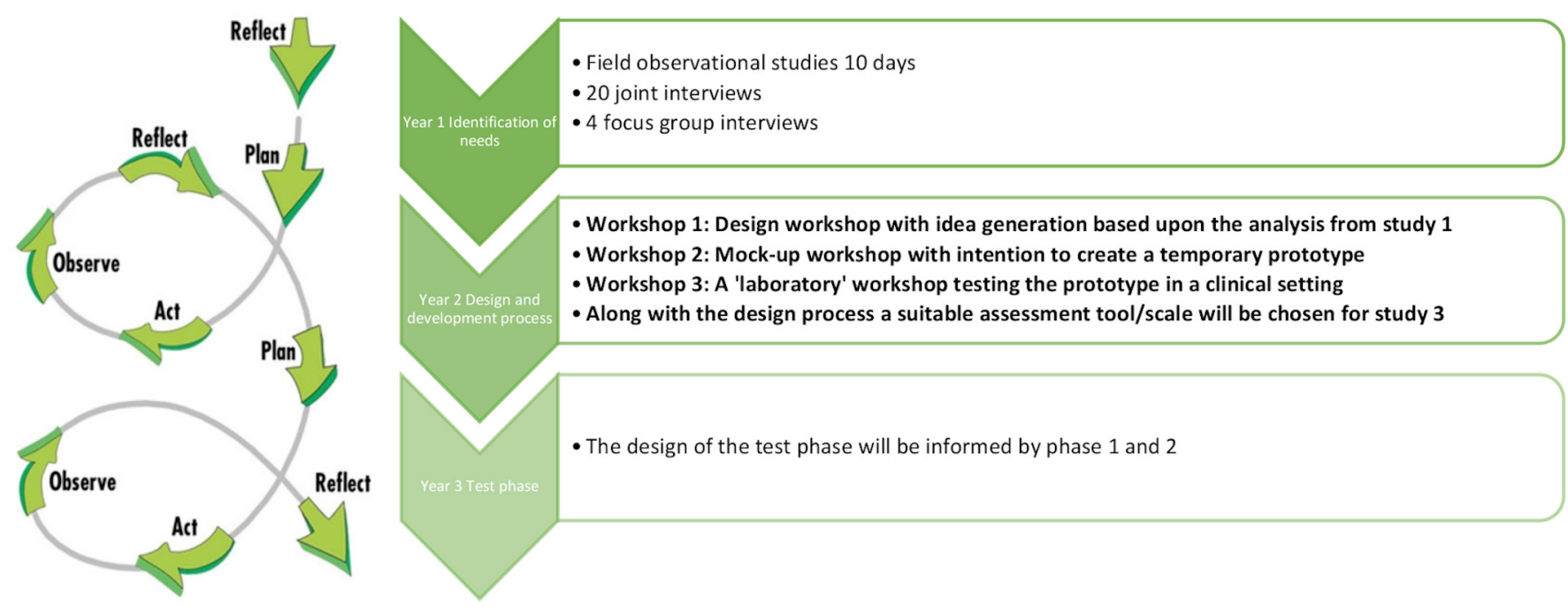

Figure 3 Iterations of phase 2: plan, act, observe and reflect. 


\section{Patient and public involvement}

The local Patient Council at OUH was consulted in the early design phase of the study, and their perspectives were taken into account. The core element of the study is built around user involvement and its strengths and limitations will be elaborated on in the discussion section.

\section{DISCUSSION}

The use of a PD provides an innovative approach through the inclusion of users across the healthcare setting. PD and its methods are very productive research approaches, directing the design of the solution to support patients' needs and organisational changes in clinical practice. ${ }^{3151}$ The participatory approach ensures stakeholder involvement and sustainability of the designed solution as it is drawn directly from patients, family members and healthcare professionals. The data will provide a strong foundation to improve patient-valued outcomes and experiences of support. Co-production and focus on future end-users are increasingly applied in designing and improving healthcare, and have shown great potential to improve the quality and value of care. ${ }^{30452}$ In our study, we base the design and development on a qualitative foundation from the two main groups of end-users; patients' /family members' and healthcare professionals' descriptions of needs and preferences. By actively involving participants, the solution will be targeted at the main issues 8 in acute care and the likelihood of actually improving family-inclusive patient outcomes will increase. We consider participant interaction to be one of our study's main strengths, enabling a deeper understanding of emergency care. Collecting data at two different sites is considered a strength, as it will ensure the national generalisability of the findings.

As our protocol is based on co-production, it may be at risk of logistical and practical challenges by gathering different stakeholders. Challenges posed by engaging healthcare professionals in workshops relate to staff resources, and this must be addressed. ${ }^{53}$ Phase 1 challenges will be to sample enough participants to be representative as the ED has a great diversity of patients with different ages, needs and diseases. Therefore, purposive sampling is chosen. Field observations may lead to irrelevant focus ${ }^{34}$ and risk of the Hawthorne effect, ${ }^{54}$ however, using an observation guide inspired by Spradley will ensure a systematic approach. ${ }^{34}$ Although it is expected that both parties (patient and family members) will actively participate in joint interviews, the advantages and disadvantages must be addressed. The main disadvantage is the risk that one of the participants may be more conversational and may overrule the other one. However, joint interviews are chosen as the authors want to explore both perspectives and create a social interaction that could bring out their experiences in a nuanced way. ${ }^{44}$ Involving participants actively in workshops and working in iterative processes will place demands regarding flexibility and willingness to change direction, if participants say so. This may be time-consuming and cost-intensive.

\section{Summary}

By focussing on co-production, this study is expected to contribute to an improved health outcome of acute illness and an improved understanding of how to support patients and family members to reach the ability to manage their situation after a short ED episode.

\section{Author affiliations}

${ }^{1}$ Department of Emergency Medicine, Odense Universitetshospital, Odense, Denmark

${ }^{2}$ Clinical Institute, University of Southern Denmark Faculty of Health Sciences,

Odense, Denmark

${ }^{3}$ Department of Orthopedics Surgery and Traumatology, Odense Universitetshospital, Odense, Denmark

${ }^{4}$ School of Nursing and Midwifery, Logan Campus, Griffith University Faculty of Health, Brisbane, Queensland, Australia

${ }^{5}$ Department of Oncology, Odense University Hospital, Odense, Denmark

Correction notice This article has been corrected since it first published. The provenance and peer review statement has been included.

Twitter Christina Østervang @C_Oestervang and Elisabeth Coyne @ Elisabethcoyne1

Contributors ATL and CØ conceived the study. ATL, CMJ, KBD, EC and CØ designed the study. $\varnothing \emptyset$ took the lead in drafting the study protocol manuscript, receiving inputs and feedback from ATL, CMJ, EC and KDB. All authors approved the final protocol manuscript.

Funding The study has received grants from The University of Southern Denmark, Odense University Hospital and the Region of Southern Denmark.

Competing interests None declared.

Patient and public involvement Patients and/or the public were involved in the design, or conduct, or reporting, or dissemination plans of this research. Refer to the Methods section for further details.

Patient consent for publication Not required.

Ethics approval The study is registered with the Danish Data Protection Agency (19/22672), and data will be stored at a logged server at Open Patient data Explorative Network (OPEN_938), Department of Clinical Research, University of Southern Denmark. The study is approved by the Regional Committees on health research Ethics for Southern Denmark (S-20192000-111).

Provenance and peer review Not commissioned; externally peer reviewed.

Data availability statement Data sharing not applicable as no data sets were generated and/or analysed for this study yet. Data sharing is not applicable as there is no data set yet.

Open access This is an open access article distributed in accordance with the Creative Commons Attribution Non Commercial (CC BY-NC 4.0) license, which permits others to distribute, remix, adapt, build upon this work non-commercially, and license their derivative works on different terms, provided the original work is properly cited, appropriate credit is given, any changes made indicated, and the use is non-commercial. See: http://creativecommons.org/licenses/by-nc/4.0/.

\section{ORCID iDs}

Christina Østervang http://orcid.org/0000-0001-5990-0167

Annmarie Touborg Lassen http://orcid.org/0000-0003-4942-6152

Charlotte Myhre Jensen http://orcid.org/0000-0002-7058-4641

Elisabeth Coyne http://orcid.org/0000-0001-8511-600X

Karin Brochstedt Dieperink http://orcid.org/0000-0003-4766-3242

\section{REFERENCES}

1 Hess EP, Grudzen CR, Thomson R, et al. Shared decision-making in the emergency department: respecting patient autonomy when seconds count. Acad Emerg Med 2015;22:856-64.

2 Elmqvist C, Frank C. Patients' strategies to deal with their situation at an emergency department. Scand J Caring Sci 2015;29:145-51.

3 Wåhlin I. Empowerment in critical care - a concept analysis. Scand J Caring Sci 2017;31:164-74. 
4 Hsiao P-R, Redley B, Hsiao Y-C, et al. Family needs of critically ill patients in the emergency department. Int Emerg Nurs 2017;30:3-8.

5 Olgers TJ, Dijkstra RS, Drost-de Klerck AM, et al. The ABCDE primary assessment in the emergency department in medically ill patients: an observational pilot study. Neth J Med 2017;75:106-11.

6 Nielsen LM, Gregersen Østergaard L, Maribo T, et al. Returning to everyday life after discharge from a short-stay unit at the Emergency Department-a qualitative study of elderly patients' experiences. Int J Qual Stud Health Well-being 2019;14:1563428.

7 Scott I, Vaughan L, Bell D. Effectiveness of acute medical units in hospitals: a systematic review. Int J Qual Health Care 2009;21:397-407.

8 Stevens L, Fry M, Browne M, et al. Fast track patients' satisfaction, compliance and confidence with emergency department discharge planning. Australas Emerg Care 2019;22:87-91.

9 Christiansen T, Vrangbæk K. Hospital centralization and performance in Denmark-Ten years on. Health Policy 2018;122:321-8.

10 Frank C, Asp M, Dahlberg K. Patient participation in emergency care - a phenomenographic analysis of caregivers' conceptions. J Clin Nurs 2009;18:2555-62.

11 Forsgärde E-S, From Attebring M, Elmqvist C. Powerlessness: dissatisfied patients' and relatives' experience of their emergency department visit. Int Emerg Nurs 2016;25:32-6.

12 Kompetencecenter for Patientoplevelser, Landsdækkende Undersøgelse AF Patientoplevelser, 2017

13 Locock L, Boylan A-M, Snow R, et al. The power of symbolic capital in patient and public involvement in health research. Health Expect 2017:20:836-44.

14 INVOLVE, I.N. What is public involvement in research? 20182018 [cited 2019 18.02]; Available from: What is public involvement in research? 2018

15 Feinberg LF. Moving toward Person- and Family-Centered care. Public Policy \& Aging Report 2014;24:97-101.

16 Muntlin A, Carlsson M, Gunningberg L. Barriers to change hindering quality improvement: the reality of emergency care. $J$ Emerg Nurs 2010;36:317-23.

17 Mixon AS, Goggins K, Bell SP, et al. Preparedness for hospital discharge and prediction of readmission. $J$ Hosp Med 2016;11:603-9.

18 Vaillancourt S, Seaton MB, Schull MJ, et al. Patients' Perspectives on Outcomes of Care After Discharge From the Emergency Department: A Qualitative Study. Ann Emerg Med 2017;70:648-58.

19 Hoffmann E, Olsen PR. Like an ACE up the sleeve: an interview study of nurses' experiences of the contact with relatives in a somatic emergency ward. Scand J Caring Sci 2018;32:1207-14.

20 Lavesen M, Ladelund S, Frederiksen AJ, et al. Nurse-initiated telephone follow-up on patients with chronic obstructive pulmonary disease improves patient empowerment, but cannot prevent readmissions. Dan Med J 2016;63.

21 Biese KJ, Busby-Whitehead J, Cai J, et al. Telephone follow-up for older adults discharged to home from the emergency department: a pragmatic randomized controlled trial. J Am Geriatr Soc 2018;66:452-8.

22 Hoek AE, Anker SCP, van Beeck EF, et al. Patient discharge instructions in the emergency department and their effects on comprehension and recall of discharge instructions: a systematic review and meta-analysis. Ann Emerg Med 2020;75:435-44.

23 Rosted E, Poulsen I, Hendriksen C, et al. Testing a two step nursing intervention focused on decreasing rehospitalizations and nursing home admission post discharge from acute care. Geriatr Nurs 2013;34:477-85.

24 Flynn D, Knoedler MA, Hess EP, et al. Engaging patients in health care decisions in the emergency department through shared decision-making: a systematic review. Acad Emerg Med 2012;19:959-67.

25 Melnick ER, Probst MA, Schoenfeld E, et al. Development and testing of shared decision making interventions for use in emergency care: a research agenda. Acad Emerg Med 2016;23:1346-53.

26 Lisby M, Klingenberg M, Ahrensberg JM, et al. Clinical impact of a comprehensive nurse-led discharge intervention on patients being discharged home from an acute medical unit: randomised controlled trial. Int J Nurs Stud 2019;100:103411.

27 Simonsen J, Robertson T. Routledge international Handbook of participatory design. Routledge, 2012.
28 Wright, Leahey M. Nurses and families a guide to family Assesment and intervention. 6th ed. F.A. Davis Company, 2013.

29 Craig Pet al. Medical Research Council complex interventions guidance, 2008.

30 Jensen CM, Overgaard S, Wiil UK, et al. Bridging the gap: a user-driven study on new ways to support self-care and empowerment for patients with hip fracture. SAGE Open Med 2018;6:205031211879912.

31 Clemensen J, Rothmann MJ, Smith AC, et al. Participatory design methods in telemedicine research. $J$ Telemed Telecare 2017;23:780-5.

32 Clemensen J, Larsen SB, Kyng M, et al. Participatory design in health sciences: using cooperative experimental methods in developing health services and computer technology. Qual Health Res 2007:17:122-30.

33 Coster S, Watkins M, Norman IJ. What is the impact of professional nursing on patients' outcomes globally? an overview of research evidence. Int J Nurs Stud 2018;78:76-83.

34 Spradley J. Participant observation. USA: Waveland Press, 1990.

35 Voltelen B, Konradsen H, Østergaard B. Ethical considerations when conducting joint interviews with close relatives or family: an integrative review. Scand J Caring Sci 2018;32:515-26.

36 Family Focused Healthcare Research Center. 2020 09.03.2020 [cited 2020 09.03]; Available from. Available: https://www.sdu.dk/en/om sdu/institutter_centre/klinisk_institut/forskning/forskningsenheder/ face

37 DEPT. User Manuel Danish Emergency Process Triage - DEPT. Available: http://deptriage.dk/brugervejledning/

38 Sundhedsstyrelsen, Danske Regioner, and Sundheds OG Fldreministeriet, de danske akutmodtagelser. Statusrapport 2016, 2017

39 Moser A, Korstjens I. Series: practical guidance to qualitative research. Part 3: sampling, data collection and analysis. Eur J Gen Pract 2018:24:9-18.

40 Green J, Thorogood N. Qualitative methods for health research. 3rd ed. Sage, 2014.

41 Østervang C. Patient Rounds With Video-Consulted RelativesQualitative Study on Possibilities and Barriers From the Perspective of Healthcare Providers.pdf $>, 2019$

42 Vestergaard LV, Østervang C, Danbjørg DB, et al. Video-Based patient rounds for caregivers of patients with cancer. Oncol Nurs Forum 2019:46:485-92.

43 Kvale S, Brinkmann S. Interview: introduktion til et håndværk. Kbh: Hans Reitzel, 2009.

44 Halkier B. Fokusgrupper, in Kvalitative metoder: en grundbog. København: Hans Reitzel, 2010.

45 Coyne E, Grafton E, Reid A. Strategies to successfully recruit and engage clinical nurses as participants in qualitative clinical research. Contemp Nurse 2016;52:669-76.

46 Malterud K. Systematic text condensation: a strategy for qualitative analysis. Scand J Public Health 2012;40:795-805.

47 O'Brien BC, Harris IB, Beckman TJ, et al. Standards for reporting qualitative research: a synthesis of recommendations. Acad Med 2014;89:1245-51.

48 Nielsen C, Agerskov H, Bistrup C, et al. User involvement in the development of a telehealth solution to improve the kidney transplantation process: a participatory design study. Health Informatics J 2020;26:1237-52.

49 Sykepleiernes Samarbeid i Norden Northern Nurses' Federation, Ethical guidelines for nursing research in the Nordic countries, 2003

50 OPEN. Open Patient data Explorative Network Clinical Institute University of Southern Denmark, 2020. Available: https://www.sdu. $\mathrm{dk} / \mathrm{da} / \mathrm{ki} /$ open

51 Joshi SG, Bratteteig T. Designing for prolonged mastery. on involving old people in participatory design. Scand J Inf Syst 2016;28.

52 Trettin B, Feldman SR, Andersen F, et al. A changed life: the life experiences of patients with psoriasis receiving biological treatment. Br J Dermatol 2020;183:516-23.

53 Danbjørg DB, Wagner L, Kristensen BR, et al. Intervention among new parents followed up by an interview study exploring their experiences of telemedicine after early postnatal discharge. Midwifery 2015;31:574-81.

54 Mostafazadeh-Bora M. The Hawthorne effect in observational studies: threat or opportunity? Infect Control Hosp Epidemiol 2020;1. 